\title{
Manejo y seguimiento nutricional de los pacientes con cirugía bariátrica en Colombia
}

\author{
Medical approach and nutritional follow-up of patients undergoing \\ bariatric surgery in Colombia
}

\author{
Juan Sebastián Ballesteros', Iván Darío Hernández¹.
}

Recibido: 15 de diciembre 2017. Aceptado para publicación: 1 de marzo 2018 https://doi.org/10.35454/rncm.v1n1.074

\begin{abstract}
Resumen
Introducción: la cirugía bariátrica se asocia a alteraciones metabólicas, desnutrición proteico-calórica y deficiencias nutricionales cuando no se hace un manejo y un seguimiento médico nutricional adecuado del paciente por parte de equipos interdisciplinarios. Hasta el año 2012 Colombia no contaba con una guía de manejo propia y se desconocía la práctica actual de los grupos de cirugía bariátrica. Por lo tanto, este estudio tuvo como objetivo conocer el seguimiento y manejo nutricional que realizan los médicos y nutricionistas involucrados en la cirugía bariátrica en el país.

Metodología: se trata de un estudio descriptivo tipo encuesta. A 30 médicos o nutricionistas de cirugía bariátrica se les aplicó una herramienta validada por expertos y basada en cuatro consensos internacionales.

Resultados: se obtuvo la participación de 11 de los 30 profesionales encuestados, siete de los cuales hacían parte de grupos interdisciplinarios. El procedimiento más frecuentemente practicado fue la manga gástrica. Los encuestados consideraron que un IMC de $35 \mathrm{~kg} / \mathrm{m}^{2}$ o más con enfermedades concomitantes era indicación para cirugía bariátrica; uno la consideró indicada en caso de IMC mayor de $30 \mathrm{~kg} / \mathrm{m}^{2}$ con enfermedades concomitantes $\mathrm{y}$, otro, en caso de IMC mayor de $35 \mathrm{~kg} / \mathrm{m}^{2}$ sin enfermedades concomitantes. Cinco de los encuestados refirieron que el tiempo de tratamiento antes de la cirugía fue menor de un mes. Todos prescribieron suplementos vitamínicos postoperatorios, aunque la cantidad y el tipo de suplemento varió de un profesional a otro. Los indicadores utilizados para el seguimiento del éxito de la cirugía fueron, en su mayoría, el IMC y el porcentaje de pérdida de peso. El seguimiento postoperatorio fue diferente según el tipo de cirugía; seis lo iniciaron en la primera semana.

Conclusión: este estudio permite observar una gran heterogeneidad en el manejo y seguimiento nutricionales en la cirugía bariátrica, por parte de los profesionales encuestados. Con el auge de este método para bajar de peso y reducir enfermedades concomitantes, es necesario fomentar la creación de grupos interdisciplinarios de excelencia regidos por una guía o consenso nacional.
\end{abstract}

Palabras clave: cirugía bariátrica, desnutrición, obesidad, vitaminas, proteína, Índice de Masa Corporal.

1 Universidad El Bosque, Bogotá, D.C., Colombia

\section{Summary}

Introduction: The bariatric surgery is associated with metabolic disorders, protein calorie malnutrition and nutritional deficiencies without proper management and patient follows up by interdisciplinary teams. Until 2012, Colombia did not have its own guidelines and ignored the current practice of bariatric surgery groups. Therefore, this study aimed to determine what follow up and nutritional approach surgeons and nutritionists have made in the country.

Methodology: A quantitative descriptive study, survey type, was carried out. We surveyed 30 bariatric dieticians or surgeons using a questionnaire validated by experts, based on four international consensuses.

Results: We received only 11 replies of the 30 questionnaires, seven professionals belong to interdisciplinary groups. The most frequently performed procedure was gastric sleeve (7 cases). All the surveyed professionals considered a BMl equal to or greater than $35 \mathrm{~kg} / \mathrm{m}^{2}$ with any comorbidity as an indication for bariatric surgery; one reported that it should be carried out if BMI was greater than $30 \mathrm{~kg} / \mathrm{m}^{2}$ with any comorbidity, and another professional said that it should be done if BMI was greater than $35 \mathrm{~kg} /$ $\mathrm{m}^{2}$ without any comorbidity. The time of treatment before the surgery was less than one month in for five respondents. Two of the respondents did not ordered any type of diet preoperatively. The total respondents prescribed postoperative vitamin supplements; it varied the amount and type of supplement. The weight of reference for monitoring the success of surgery was mostly $\mathrm{BMI}$ and percentage of weight loss. The postoperative follow-up varied according to the type of surgery, six began control visits in the first week.

Conclusion: This study allows us to observe a large heterogeneity in the nutritional follow up and control by the professionals surveyed. It is clear that nowadays, with the rise of this method for weight losing and reducing comorbidities, it is necessary to encourage excellence in interdisciplinary groups governed by national guidance or consensus.

Keywords: Bariatric surgery; Malnutrition; Obesity; Vitamins; Protein; Body Mass Index (BMI). 


\section{INTRODUCCIÓN}

La obesidad ha alcanzado proporciones epidémicas en el ámbito mundial y cada año mueren como mínimo 2,6 millones de personas a causa de la obesidad o sobrepeso. Aunque anteriormente se consideraba un problema confinado a los países de altos ingresos, en la actualidad la obesidad también es prevalente en los países de ingresos bajos y medianos ${ }^{(1)}$.

En Colombia, según la encuesta ENSIN sobre la situación nutricional de la población colombiana (y sus determinantes socioeconómicos) en 2015 se observó un incremento en el exceso de peso en todos los grupos poblacionales. En particular, en los adultos el aumento es importante, de $51,2 \%$ en 2010 a 56,4 \% en 2015. Más de la mitad de la población adulta en Colombia presenta sobrepeso $(37,7 \%)$ u obesidad $(18,7 \%)$. Es de resaltar que la obesidad es más frecuente en las mujeres $(22,4 \%)$ que en los hombres (14,4\%). Solo la mitad de los adultos cumple con las recomendaciones de la Organización Mundial de la Salud de realizar 150 minutos semanales de actividad física moderada o 75 minutos semanales de actividad vigorosa o fuerte ${ }^{(2)}$.

La obesidad se asocia a incremento de la morbilidad y la mortalidad ${ }^{(3)}$. Este aumento en la morbilidad está relacionado con resistencia a la insulina, diabetes, dislipidemia e hipertensión, afecciones de alta prevalencia a tanto en el país como en el ámbito mundial ${ }^{(4)}$. Se estima que $90 \%$ de los pacientes con diabetes mellitus de tipo 2 son obesos. La obesidad ha sido incluida, por la American Heart Association (AHA) como uno de los mayores factores de riesgo para desarrollar enfermedad coronaria ${ }^{(5)}$. Existe, además, correlación entre obesidad y distintos tipos de cáncer, entre los que se incluyen el de colon, esófago, páncreas, endometrio, mama y próstata ${ }^{(6)}$.

Para disminuir la obesidad y los problemas asociados se han empleado varios tratamientos, entre los que se encuentran el manejo nutricional, el farmacológico y el quirúrgico. La cirugía bariátrica abarca aquellos procedimientos quirúrgicos que son practicados para disminuir el peso y las enfermedades concomitantes asociadas ${ }^{(7,8)}$, está indicada como tratamiento en: a. pacientes con índice de masa corporal (IMC) mayor de $35 \mathrm{~kg} / \mathrm{m}^{2}$ con complicaciones relacionadas con la obesidad, b. con un IMC mayor de $40 \mathrm{~kg} / \mathrm{m}^{2}$, c. que han fallado con las modificaciones del estilo de vida o la intervención farmacológica ${ }^{(9)}$.

Existen tres tipos de técnicas: 1) restrictivas, que reducen el tamaño del estómago mediante la formación de un reservorio adyacente al cardias y comunicado por un orificio que permite un lento vaciado gástrico, lo cual produce sensación de saciedad; entre ellas están la manga y la banda gástrica; 2) malabsortivas, que limitan la absorción de los alimentos ingeridos, con lo que se disminuye la cantidad de nutrientes que pasan a la circulación portal; entre estas está la derivación yeyuno-ileal; y 3 ) mixtas, que combinan la reducción gástrica con mala absorción intestinal, por ejemplo, la derivación bilio-pancreática y la derivación gástrica por gastro-yeyunostomía en Y de Roux ${ }^{(10)}$.

Con estas técnicas se inducen cambios gastrointestinales anatómicos y funcionales que provocan reducción de la ingesta alimentaria o una absorción de nutrientes inadecuada. Este tipo de cirugía ha venido en aumento, a tal punto que, en la actualidad, Colombia es el segundo país en Suramérica con mayor número de cirugías bariátricas después de Brasil, con más de 7.000 intervenciones por año (datos sin publicar).

En los últimos años, el índice de intervenciones ha ido en aumento, no solo para disminuir la tasa de complicaciones y enfermedades asociadas a la obesidad, sino también, por estética y factores psicosociales que afectan el normal desarrollo de la personalidad ${ }^{(11)}$. Hoy en día, la cirugía bariátrica se considera el tratamiento más efectivo para perder peso, pues el mantenimiento de la pérdida de peso a cinco años es mejor comparado con otros tratamientos, como la dietoterapia y los tratamientos farmacológicos, así como la mejoría de las enfermedades concomitantes asociadas ${ }^{(12,13)}$.

Aunque la cirugía bariátrica se practica con éxito desde los años 50, en las últimas dos décadas los procedimientos han progresado, haciéndose más seguros y efectivos. Sin embargo, existen complicaciones a mediano y largo plazo, entre las que se encuentran alteraciones metabólicas, desnutrición proteico-calórica y deficiencia de micronutrientes (Tabla 1$)^{(14)}$. La mayoría de los pacientes desarrolla alguna deficiencia nutricional, lo que justifica el suplemento de vitaminas y minerales en todos ellos. Este déficit nutricional es proporcional a la longitud del área de absorción y al porcentaje de pérdida de peso. Los niveles bajos de hierro, vitamina $\mathrm{B}_{12}$, vitamina $\mathrm{D}$ y calcio son predominantes luego de la derivación gástrica por gastro-yeyunostomía en Y de Roux. Las deficiencias de proteína y de vitaminas liposolubles son predominantes en la derivación bilio-pancreática, mientras que la de tiamina es común en pacientes con vómito frecuente. Debido a que las deficiencias progresan con el tiempo, el paciente debe ser observado de manera regular $\mathrm{y}$ frecuente para prevenir la desnutrición. El seguimiento y control adecuados por parte de un equipo interdisciplinario podrían prevenir la aparición de esas carencias y de mala nutrición ${ }^{(15)}$. 
Tabla 1. Prevalencia de deficiencia de micronutrientes antes y después de la cirugia(14)

\begin{tabular}{|c|c|c|}
\hline Micronutriente & $\begin{array}{c}\text { Prevalencia de } \\
\text { deficiencia de } \\
\text { micronutrientes en } \\
\text { pacientes obesos } \\
\text { antes de la cirugía } \\
\text { bariátrica }\end{array}$ & $\begin{array}{l}\text { Prevalencia de } \\
\text { deficiencia de } \\
\text { micronutrientes en } \\
\text { pacientes obesos } \\
\text { después de la } \\
\text { cirugía bariátrica }\end{array}$ \\
\hline Vitamina $\mathrm{A}$ & $14 \%$ & Hasta $70 \%$ \\
\hline Vitamina B1 & $29 \%$ & $\begin{array}{l}1 \% \text { a } 49 \% \\
\text { según el tipo de } \\
\text { cirugía y tiempo } \\
\text { postoperatorio. }\end{array}$ \\
\hline Vitamina B12 & $\begin{array}{l}2 \% \text { - } 18 \% \text { y } 6 \% \text { - } \\
30 \% \text { en pacientes } \\
\text { con inhibidores } \\
\text { de bomba de } \\
\text { protones }\end{array}$ & \begin{tabular}{|l|}
$2-5$ años \\
postoperatorios: \\
Post-RYGB $20 \%$ \\
Post-Sleve $4 \%-20 \%$
\end{tabular} \\
\hline Ácido fólico & $54 \%$ & Hasta $65 \%$ \\
\hline Vitamina D & $90 \%$ & Hasta $100 \%$ \\
\hline Vitamina $\mathrm{E}$ & $22 \%$ & No es frecuente \\
\hline Vitamina K & No hay datos & No es freceunte \\
\hline Cobre & $70 \%$ en mujeres & $\begin{array}{l}\text { Hasta } 90 \% \\
\text { posderivación } \\
\text { Post-RYGB 10-20\% }\end{array}$ \\
\hline Hierro & $45 \%$ & $\begin{array}{l}\text { Reportes de déficit } \\
\text { desde los } 3 \text { meses } \\
\text { hasta los } 10 \text { años } \\
\text { del postoperatorio. } \\
\text { Posbanda: } 14 \% \\
\text { Post-Sleeve: }<18 \% \\
\text { Post-RYGB: } 20-55 \% \\
\text { Posderivación: } \\
13 \% \text { - } 62 \% \\
\text { Switch duodenal: } \\
8 \%-50 \%\end{array}$ \\
\hline Zinc & $24 \%-28 \%$ & \begin{tabular}{|l|} 
Hasta $70 \%$ \\
posderivación \\
Post-RYGB: $40 \%$ \\
Post-Sleeve: $19 \%$ \\
Posbanda: $30 \%$
\end{tabular} \\
\hline
\end{tabular}

BPGYR: derivación gástrica por gastro-yeyunostomía en $Y$ de Roux; DBP: derivación bilio-pancreática; $\mathrm{CD}$ : cruce duodenal.

En la actualidad, existen diversos consensos que guían o pautan el manejo nutricional de estos pacientes, los cuales han sido elaborados por diferentes entidades académicas en Europa, Norteamérica, España y Argentina. En Colombia, hasta el año 2012 no existía una guía o consenso nacional ${ }^{(16)}$. La Asociación Colombiana de Nutrición Clínica publicó en 2013 el primer consenso y luego en el año 2014 la Asociación Colombiana de Obesidad y Cirugía Bariátrica publicó las guías de manejo nacionales ${ }^{(17)}$. Este estudio, llevado a cabo en 2011, tuvo como objetivo determinar el manejo y el seguimiento nutricional que reciben los pacientes sometidos a cirugía bariátrica por obesidad en el país, antes de la emisión de una guía nacional por parte de un grupo de expertos en el tema.

\section{METODOLOGÍA}

Se llevó a cabo un estudio descriptivo y cuantitativo.

Herramienta. La encuesta fue desarrollada teniendo en cuenta una revisión extensa de la literatura científica y los aspectos más importantes del manejo nutricional, según los consensos europeo, norteamericano, español y argentino, sobre nutrición en cirugía bariátrica ${ }^{(8,18-23)}$. La encuesta fue validada según criterio de expertos. Tres nutricionistas de la Asociación Colombiana de Nutrición Clínica hicieron la validación. La encuesta consistió de dos partes, como sigue.

Hoja 1. Información general sobre el grupo. Esta hoja debía ser diligenciada por la nutricionista o el cirujano. Se buscó obtener información sobre las características generales de los integrantes del grupo (si lo había) y su funcionamiento.

Hoja 2. Manejo y seguimiento nutricionales. Esta hoja debía ser diligenciada por el profesional encargado de hacer el manejo y seguimiento nutricionales, el médico o el nutricionista. Se buscó obtener información detallada sobre las características del manejo y seguimiento nutricionales, antes y después de la cirugía.

Encuestados. Se envió la encuesta por correo electrónico a 30 nutricionistas y cirujanos bariátricos, asociados o en contacto con la Asociación Colombiana de Nutrición Clínica. La recepción de la encuesta y la participación en el estudio se confirmaron vía telefónica. La participación fue voluntaria.

\section{Consideraciones éticas}

Se consideró una investigación sin riesgo, según la Resolución No 008430 del 4 de octubre de 1993, en cuanto que este estudio no implicaba ninguna intervención o modificación intencionada de las variables biológicas, fisiológicas, psicológicas o sociales de participantes en el estudio.

La información se trató con confidencialidad y se protegió la identidad de las instituciones, los médicos y nutricionistas que participaron. 


\section{RESULTADOS}

El estudio contó con la participación de 11 de los 30 profesionales encuestados, de los cuales nueve eran nutricionistas y dos cirujanos. En cuanto a la caracterización de los encuestados, se encontró que ocho estaban vinculados a instituciones privadas y tres a públicas. Se encuestaron siete profesionales que hacían parte de grupos interdisciplinarios constituidos, mientras que cuatro no. En cuanto al procedimiento más frecuentemente ejecutado por los grupos a los que pertenecían los profesionales o cirujanos (más de una respuestas era posible), se encontró que siete encuestados realizan la manga gástrica (o hacen parte de grupos que la practican), seis, la derivación gástrica por gastro-yeyunostomía en Y de Roux, y dos, el balón gástrico (Figura 1).

La mayoría de los encuestados consideró que un IMC de $35 \mathrm{~kg} / \mathrm{m}^{2}$ o más con enfermedades concomitantes era indicación para cirugía bariátrica; uno la considera indicada en caso de IMC mayor de $30 \mathrm{~kg} / \mathrm{m}^{2}$ con enfermedades concomitantes y, otro, en caso de IMC mayor de $35 \mathrm{~kg} / \mathrm{m}^{2}$ sin enfermedades concomitantes.

El tiempo de tratamiento médico nutricional antes de la cirugía fue menor de un mes para cinco encuestados, de uno a tres meses para un encuestado, y mayor de tres meses en dos de los casos; esta pregunta no fue respondida en tres ocasiones.

En cuanto al manejo preoperatorio, dos encuestados no prescriben ningún tipo de dieta. De los nueve que sí la prescriben, siete lo hacen por más de 10 días y, dos, por menos de 10 días. La consistencia de la dieta ordenada era normal para cinco, blanda para uno y líquida para tres de los encuestados. De los 11 encuestados, siete no exigen pérdida de peso preoperatoria. La metodología utilizada en el cálculo de la ingesta alimentaria, para cuatro encuestados es el recordatorio de 24 horas; cuatro utilizan otros métodos y cinco no la registran.

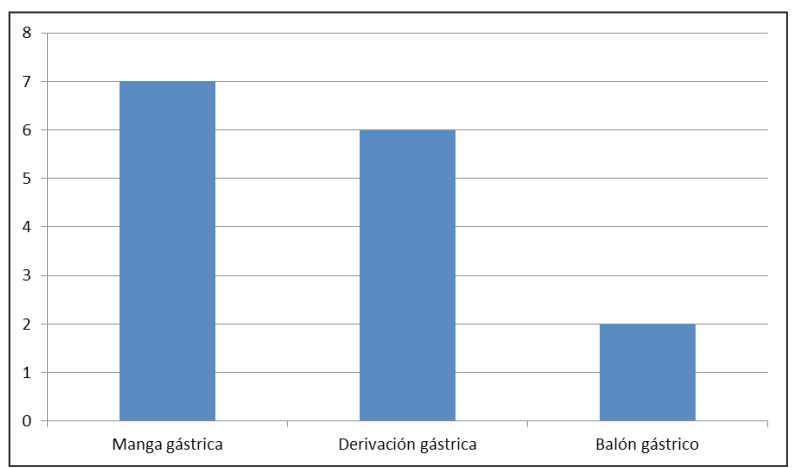

Figura 1. Tipo de procedimientos que realizan los encuestados.
El indicador de referencia para el seguimiento del éxito de la cirugía que utilizan tres de los encuestados fue el IMC, uno utiliza el porcentaje de pérdida de peso, cuatro utilizan el IMC y el porcentaje de pérdida de peso, otro utiliza el porcentaje de grasa corporal, mientras que dos no respondieron a la pregunta (Figura 2).

En cuanto al manejo nutricional postoperatorio, seis de los encuestados hacen la primera visita antes de los ocho días, cuatro, después de ocho días, y uno no lo refirió.

En cuanto a los controles nutricionales, cinco de los encuestados los llevan a cabo semestralmente luego del primer año, tres hacen más de dos controles al año, mientras que uno de los encuestados no hace controles y dos nutricionistas no respondieron a esta pregunta.

La dieta postoperatoria, en todos los casos, se hace en etapas de duración variable y de características similares, pasando de líquida a normal. La totalidad de los encuestados prescribe suplementos vitamínicos, nueve prescriben vitamina $B_{12}$; seis, hierro; nueve, calcio; siete, vitamina $\mathrm{D}$; dos, ácido fólico; ocho, proteína, y ocho, multivitamínicos (Figura 3). Sobre la actividad física, ocho de los encuestados refirieron asociarla al manejo nutricional del paciente operado, $y$ tres no lo hicieron.

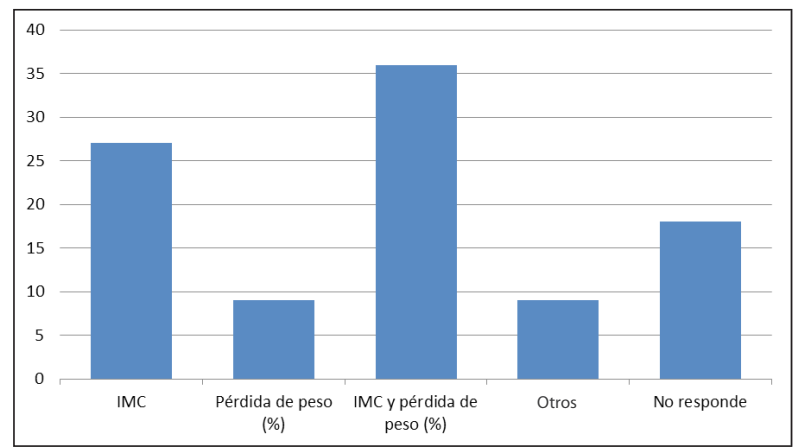

Figura 2. Métodos de seguimiento con los cuales se verifica el éxito del procedimiento. Otros: porcentaje de grasa perdido.

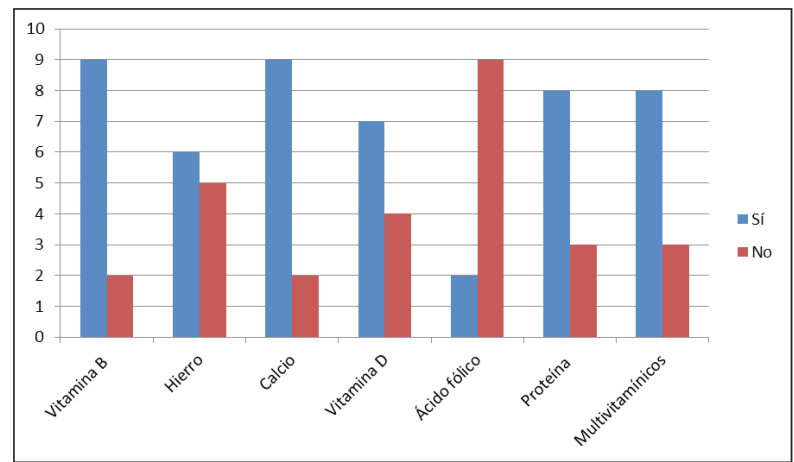

Figura 3. Suplemento vitamínico en el postoperatorio 


\section{DISCUSIÓN}

La cirugía bariátrica, a pesar de tener éxito en el mantenimiento de la pérdida de peso y en la mejoría de las enfermedades concomitantes asociadas a la obesidad, conlleva a un riesgo importante en la aparición de desnutrición proteico-calórica y carencias nutricionales. La principal recomendación para prevenir estas complicaciones es el cumplimiento del manejo y del seguimiento nutricionales adecuados por parte de un equipo interdisciplinario ${ }^{(23)}$.

Para cumplir con este objetivo, desde 2005 se crearon centros de excelencia en países como los Estados Unidos ${ }^{(24)}$. El consenso español recomienda que la evaluación del paciente con obesidad mórbida la haga un grupo interdisciplinario que incluya endocrinólogos, cirujanos, nutricionistas, anestesiólogos, psicólogos, psiquiatras, neumólogos, gastroenterólogos, radiólogos, educadores y otros especialistas que se consideren necesarios, en función de la enfermedad asociada que presente el paciente ${ }^{(8)}$. Se encontró en este estudio, que cuatro de los cirujanos y nutricionistas encuestados no hacían parte de grupos interdisciplinarios. Esto muestra la necesidad de promover la creación de un mayor número de grupos en el país.

Actualmente se indica la cirugía bariátrica en aquellos pacientes que, después de haber fracasado en reiterados planes de tratamiento médico multidisciplinario, presentan un IMC mayor de $40 \mathrm{~kg} / \mathrm{m}^{2}$, o uno entre $35 \mathrm{y}$ $40 \mathrm{~kg} / \mathrm{m}^{2}$, con alguna enfermedad asociada de relevancia médica, como hipertensión arterial, diabetes mellitus, dislipidemias, apnea del sueño o alteraciones osteoarticulares $^{(8,21,22,25)}$ (Tabla 2).

En el estudio, dos encuestados difieren sobre estas indicaciones. Uno de ellos indica la cirugía para pacientes con un IMC mayor de $30 \mathrm{~kg} / \mathrm{m}^{2}$ y, otro, con uno mayor de $35 \mathrm{~kg} / \mathrm{m}^{2}$ sin enfermedades concomitantes, lo que podría tratarse de una tendencia actual, con el desarrollo de la cirugía metabólica ${ }^{(26)}$. En efecto, la cirugía metabólica pretende tratar la diabetes de tipo 2 y otros factores de riesgo cardiovascular sin tener en cuenta el IMC. Sin embargo, aún no hay datos suficientes para recomendar la cirugía bariátrica con un IMC por debajo de $35 \mathrm{~kg} / \mathrm{m}^{2}$.

En el estudio se encontró que la principal técnica utilizada es la manga gástrica (siete encuestados) seguida de la derivación gástrica (seis encuestados) y el balón gástrico (dos encuestados), lo que concuerda con la tendencia mundial actual ${ }^{(27)}$.

La preparación nutricional preoperatoria tiene como objetivo principal lograr el descenso previo del peso, disminución del tejido adiposo visceral y el hígado graso. Esto facilita la técnica quirúrgica y mejora los tiempos quirúrgicos; además, presenta menor riesgo de complicaciones y de pérdida de sangre durante la cirugía, con una mejor recuperación posquirúrgica, menos días de hospitalización y menor riesgo de conversión a cirugía abierta ${ }^{(28-32)}$. Los estudios demuestran que la pérdida preoperatoria de peso se asocia a mayor pérdida de peso al año de la cirugía ${ }^{(30)}$. Es claro que el objetivo principal de la preparación nutricional es evaluar si el paciente va a ajustarse a un nuevo régimen de alimentación. Algunos autores recomiendan durante las dos semanas previas a la cirugía una dieta líquida completa, como una de las herramientas para mejorar la adaptación al cambio de consistencia que tendrán que experimentar durante las primeras semanas después de la cirugía y, al mismo tiempo, para evaluar la motivación del paciente y su observancia de las indicaciones ${ }^{(21,33)}$. Sin embargo, esto es objeto de controversia. En el estudio, dos de los profesionales no prescribían dieta preoperatoria. De los nueve encuestados que sí lo hacían, solamente tres lo hacían con consistencia líquida.

El tiempo de seguimiento preoperatorio es importante para educar en temas como la nutrición y evaluar el compromiso y la observancia del tratamiento. El consenso argentino afirma que cada equipo profesional tratante debe evaluar cuánto tiempo requiere cada paciente para la preparación. La recomendación en este consenso es efectuar un seguimiento de tres meses antes de la fecha de cirugía, con un mínimo de una consulta al mes, aumentando la frecuencia en las últimas semanas antes de la cirugía. Se debe tener en cuenta el tiempo para lograr la disminución de $10 \%$ del exceso de peso y el grado de urgencia médica de la cirugía ${ }^{(28,29,34)}$. El estudio mostró que solo dos de los encuestados hace un seguimiento previo mayor o igual a tres meses, seis acostumbran un seguimiento menor de tres meses, $y$ cinco lo hacen por un periodo menor de un mes, por lo que en la mayoría de los casos no hay un tiempo ideal recomendado para el seguimiento preoperatorio, según el consenso antes nombrado. Además, siete de los encuestados no exigen pérdida de peso preoperatoria.

$\mathrm{El}$ indicador de referencia para el seguimiento del éxito de la cirugía es objeto de controversia ${ }^{(35)}$. En la actualidad se utilizan, principalmente, el IMC, el porcentaje de sobrepeso perdido y el porcentaje de pérdida de peso, según el peso de base ${ }^{(36)}$. El término "sobrepeso o exceso de peso" se refiere a la diferencia entre el peso real y el peso "ideal" de un individuo dado. Una pérdida promedio de $50 \%$ de exceso de peso en cinco años se 
Tabla 2. Indicación de cirugía, peso de referencia y seguimiento según consensos internacionales.

\begin{tabular}{|c|c|c|c|c|}
\hline & $\begin{array}{c}\text { Consenso europeo } \\
2007^{(19)}\end{array}$ & $\begin{array}{c}\text { Consenso } \\
\text { estadounidense 2009, } \\
2016^{(29,40)} \\
\end{array}$ & $\begin{array}{l}\text { Consenso } \\
\text { argentino } 2010^{(21)}\end{array}$ & $\begin{array}{c}\text { Consenso español } \\
200^{(48)}\end{array}$ \\
\hline $\begin{array}{c}\text { Manejo } \\
\text { interdisciplinario }\end{array}$ & + & + & + & + \\
\hline $\begin{array}{l}\text { Indicaciones de } \\
\text { cirugía bariátrica }\end{array}$ & $\begin{array}{l}\text { IMC } \geq 40 \mathrm{~kg} / \mathrm{m}^{2} \\
\mathrm{o} \geq 35 \mathrm{~kg} / \mathrm{m}^{2} \text { con } \\
\text { enfermedades } \\
\text { concomitantes } \\
\text { mayores asociadas, } \\
\text { que pueden mejorar } \\
\text { tras la pérdida de peso }\end{array}$ & $\begin{array}{l}\mathrm{IMC} \geq 40 \mathrm{~kg} / \mathrm{m}^{2} \\
\mathrm{o} \geq 35 \mathrm{~kg} / \mathrm{m}^{2} \text { con } \\
\text { enfermedades } \\
\text { concomitantes mayores } \\
\text { asociadas, que pueden } \\
\text { mejorar tras la pérdida } \\
\text { de peso }\end{array}$ & $\begin{array}{l}\text { IMC }>60 \mathrm{~kg} / \mathrm{m}^{2} \text { y pacientes } \\
\text { con IMC entre } 35 \text { y } 40 \mathrm{~kg} / \mathrm{m}^{2} \\
\text { con alto riesgo quirúrgico o } \\
\text { de avanzada edad }\end{array}$ & $\begin{array}{l}\mathrm{IMC} \geq 40 \mathrm{~kg} / \mathrm{m}^{2} \mathrm{o} \\
\geq 35 \mathrm{~kg} / \mathrm{m}^{2} \text { con } \\
\text { enfermedades } \\
\text { concomitantes } \\
\text { mayores asociadas, } \\
\text { que pueden mejorar } \\
\text { tras la pérdida de } \\
\text { peso }\end{array}$ \\
\hline \begin{tabular}{l|} 
Peso referente \\
para medir el \\
éxito de la cirugía
\end{tabular} & & IMC, \% PP, PSPP & $\%$ IMC & $\begin{array}{l}\text { IMC o \% del exceso } \\
\text { de IMC perdido }\end{array}$ \\
\hline $\begin{array}{l}\text { Seguimiento de } \\
\text { cirugía restrictiva }\end{array}$ & $\begin{array}{l}\text { Primer año: cada tres } \\
\text { meses, iniciando un } \\
\text { mes después, hasta } \\
\text { lograr la meta en } \\
\text { pérdida de peso; } \\
\text { después, continuar a } \\
\text { intervalos de máximo } \\
\text { un año. }\end{array}$ & $\begin{array}{l}\text { Según enfermedades } \\
\text { concomitantes } \\
\text { nutricionales o } \\
\text { metabólicas. }\end{array}$ & $\begin{array}{l}\text { Primera consulta: una } \\
\text { semana después de la cirugía. } \\
\text { Segunda consulta: a los } 14 \\
\text { días. Tercera consulta: al mes. } \\
\text { Después, una vez cada } \\
\text { mes hasta cumplir el } \\
\text { año. Segundo año: } \\
\text { cada tres meses hasta } \\
\text { finalizar el segundo año. } \\
\text { Después bianual, según el } \\
\text { cumplimiento. } \\
\end{array}$ & $\begin{array}{l}\text { Visitas en los meses } \\
1,3,6,12,18 \text { y } 24 \\
\text { A partir del segundo } \\
\text { año, anualmente. }\end{array}$ \\
\hline $\begin{array}{l}\text { Seguimiento } \\
\text { de cirugía } \\
\text { malabsortiva }\end{array}$ & $\begin{array}{l}\text { Primer año: cada tres } \\
\text { meses, iniciando un } \\
\text { mes después. Segundo } \\
\text { año: cada tres meses y } \\
\text { después, anualmente. }\end{array}$ & $\begin{array}{l}\text { Según enfermedades } \\
\text { concomitantes } \\
\text { nutricionales o } \\
\text { metabólicas }\end{array}$ & $\begin{array}{l}\text { Igual que el seguimiento } \\
\text { de los procedimientos } \\
\text { restrictivos }\end{array}$ & $\begin{array}{l}\text { Visitas en los meses } \\
1,3,6,12,18 \text { y } 24 \text {. } \\
\text { A partir del segundo } \\
\text { año, anualmente. }\end{array}$ \\
\hline
\end{tabular}

\% PP: porcentaje de pérdida de peso; PSPP: porcentaje de sobrepeso perdido.

puede considerar un éxito, aunque esto en general varía según el tipo, la agresividad y la complejidad de la cirugía. Sin embargo, Karmali et al. recomiendan el uso del porcentaje de pérdida de peso frente a los otros métodos, con el principal argumento de que el límite de peso normal de $25 \mathrm{~kg} / \mathrm{m}^{2}$ es arbitrario y los beneficios sobre las enfermedades concomitantes se logran antes de obtener el "peso ideal” (37). Además, lograr un IMC de $25 \mathrm{~kg} / \mathrm{m}^{2}$ o menor es imposible o insostenible para la gran mayoría de los pacientes ${ }^{(38)}$. El consenso español y el argentino utilizan el porcentaje de sobrepeso perdido (Tabla 2). En este estudio se encontró que los encuestados utilizan principalmente el IMC y el porcentaje de pérdida de peso $y$, ninguno, el porcentaje de sobrepeso perdido.

Según el consenso argentino, el método más apropiado para hacer una anamnesis alimentaria depende del grado de precisión buscado por el profesional y del tiempo disponible para la consulta. Ante las dificultades que implica la correcta realización de un diario dietético de siete días (método de referencia), se recomienda el recordatorio de 24 horas, por ser el método más utilizado para estimar la ingesta reciente de un individuo, debido a su practicidad y sencillez. En el estudio, solo cuatro encuestados utilizaron el método de recordatorio de 24 horas.

Las modificaciones en la anatomía digestiva y los hábitos alimenticios pueden conducir a alteraciones en el estado nutricional, como una nutrición proteicoenergética inadecuada, o al déficit selectivo de algunos micronutrientes ${ }^{(39,40)}$. Además, la cirugía puede exacerbar algunas deficiencias preexistentes. Los pacientes sometidos a cirugía bariátrica, independientemente de la técnica quirúrgica utilizada, necesitarán seguimiento y apoyo nutricional para prevenir deficiencias nutricionales $\mathrm{u}$ otras 
complicaciones metabólicas posquirúrgicas ${ }^{(31,4142)}$. Entre los más comunes, se encuentra el déficit de ácido fólico, de vitamina $B_{12} y$ de minerales. El ácido fólico y la vitamina $B_{12}$ son esenciales en la síntesis de $A D N$; su déficit causa anemia megaloblástica; $9 \%$ a $35 \%$ de los pacientes sometidos a cirugía bariátrica presentan carencia de ácido fólico. En un estudio con derivación bilio-pancreática, se evidenció déficit de esta vitamina en $21 \%$ de los intervenidos. Con respecto a la vitamina $\mathrm{B}_{12}$, esta requiere el factor intrínseco para su absorción, al cual se une para ser absorbida en el íleon. En pacientes con derivación gástrica, los factores que propician la carencia de esta vitamina son la ingesta limitada de proteínas debido a la intolerancia y la insuficiencia en la secreción de factor intrínseco ${ }^{(14)}$. La deficiencia de hierro es común en pacientes con derivación gástrica y derivación biliopancreática, causando en consecuencia, anemia ferropénica en esta población. Los pacientes con derivación gástrica presentan insuficiencia de hierro hasta cinco años después de la cirugía (Tabla 1) ${ }^{(15)}$.

Con respecto a las vitaminas liposolubles, se ha observado carencia de todas ellas; la más importante es la de vitamina $\mathrm{D}$, causada por disminución de las sales biliares, en especial, en casos de derivación bilio-pancreática. Esta deficiencia conlleva a hipocalcemia, hiperparatiroidismo secundario y, posteriormente, a osteoporosis y osteomalacia. No existen estudios controlados disponibles que determinen la dosis ni la duración del suplemento en las cirugías restrictivas, malabsortivas o mixtas. Se recomienda continuar con el suplemento y la evaluación de posibles déficits vitamínicos en forma indefinida, en casos de cirugía malabsortiva ${ }^{(8,21-23)}$ (Tabla 1). Según el consenso argentino, el multivitamínico debe aportar el $200 \%$ de la necesidad diaria en la gastrectomía vertical en manga, según las recomendaciones diarias (Recommended Daily Allowance, RDA) y, el $100 \%$, en la banda gástrica ajustable. La ASMBS recomienda medir de rutina, en el preoperatorio, la tiamina (B1), la cobalamina (B12), ácido fólico, hierro, Vitamina D y calcio, Zinc y cobre ${ }^{(31)}$. En el postoperatorio, además se recomienda medir Vitamina A, E y K.

La desnutrición proteica sigue siendo la complicación más grave por falta de macronutrientes, asociada a los procedimientos malabsortivos ${ }^{(23)}$. Se ha descrito con la derivación gástrica y con la derivación biliopancreática. En algunos estudios se ha reportado esta complicación en $13 \%$ de los pacientes obesos dos años después de una derivación gástrica por gastroyeyunostomía en Y de Roux con, al menos $150 \mathrm{~cm}$ de rama distal, en $5 \%$ de los pacientes con menos de 150 cm de rama distal ${ }^{(43,44)}$ y en 3 a $18 \%$ después de la derivación bilio-pancreática ${ }^{(45,46)}$. En otros estudios se ha encontrado solo $0 \%$ a $6 \%$ de incidencia de deficiencia de proteína después de una derivación gástrica por gastro-yeyunostomía en Y de Roux, hasta 43 meses después de la operación ${ }^{(47)}$. La desnutrición proteica provoca una tasa de hospitalización de $1 \%$ por año, después de los procedimientos de malabsorción y conduce a una morbilidad significativa ${ }^{(48)}$. No existe información sobre cuánto debe ser el suplemento diario de proteína. El consenso de la American Association of Endocrine Surgeons, recomienda un suplemento diario de $60 \mathrm{~g}$ a $120 \mathrm{~g}$ de proteína en todos los pacientes para mantener la masa magra, durante la pérdida de peso y a largo plazo. Esto es particularmente importante en aquellos pacientes sometidos a procedimientos malabsortivos con el fin de prevenir la desnutrición proteica y sus efectos. Cuando se presenta la desnutrición proteica se observa en general, de tres a seis meses después de la cirugía y es ampliamente atribuida al desarrollo de intolerancias a los alimentos ricos en proteína ${ }^{(23,41)}$. Esta encuesta reveló que el suplemento proteico no es una práctica de rutina, pues solo ocho de los encuestados la prescriben.

Los consensos europeo, norteamericano, argentino y español afirman que los pacientes sometidos a cirugía bariátrica, cualquiera que sea la técnica quirúrgica empleada, necesitan un seguimiento nutricional especializado, para evitar complicaciones quirúrgicas, déficits nutricionales $\mathrm{u}$ otras complicaciones médicas ${ }^{(8,21)}$. La dieta posquirúrgica debe proporcionar todos los nutrientes al paciente con obesidad mórbida recién intervenido, y también en los meses siguientes, satisfaciendo, por lo menos las cantidades mínimas diarias recomendadas, con un aporte de volumen reducido. Las recomendaciones nutricionales pueden variar según el tipo de intervención y se deben hacer por etapas. En este estudio, cinco de los encuestados especificaron el tipo de dieta según el procedimiento bariátrico y los seis restantes no lo hicieron. En cuanto a los controles postoperatorios, se recomienda evaluar la situación clínico-nutricional a los 3, 6, 9, 12, 18 y 24 meses después de la cirugía y, luego, cada año.

$\mathrm{Al}$ parecer, los pacientes gravemente obesos que se convierten en activos después de la cirugía bariátrica, logran mayores pérdidas de peso y mejoría en la calidad de vida, que aquellos que permanecen inactivos. Las sesiones cortas de ejercicio (de tres a cinco minutos) pueden ser apropiadas poco después de la cirugía bariátrica, en especial cuando se repiten durante el día ${ }^{(49)}$. 
La duración del ejercicio debe aumentarse poco a poco según su tolerancia. A los pacientes que sufren una artropatía, se les puede recomendar modalidades de ejercicio como la bicicleta estática o un ergómetro reclinado. Más recientemente, la International Association for the Study of Obesity (IASO) declaró que: "Hay pruebas convincentes de que la prevención de la recuperación de peso en individuos obesos requiere 60 a 90 minutos diarios de actividad de intensidad moderada o menores cantidades de actividad vigorosa" ${ }^{(50)}$. Los resultados de la presente encuesta muestran que tres de los encuestados no asocian la actividad física al manejo nutricional del paciente operado.

La principal limitación de este estudio fue la baja participación de los encuestados. Desde el punto de vista de los autores, esto puede explicarse por el temor a ser evaluados, a pesar de garantizarse el mantenimiento del anonimato en las encuestas. Se contactaron principalmente profesionales vinculados a la Asociación Colombiana de Nutrición Clínica (asociación interdisciplinaria), ya que no existe un registro nacional de profesionales que atiendan pacientes sometidos a cirugía bariátrica.

En conclusión, en una pequeña muestra de cirujanos y nutricionistas que manejan la cirugía bariátrica, este estudio permitió observar heterogeneidad en el manejo y el seguimiento nutricionales. Esto se observa en especial en el manejo preoperatorio, la administración de suplementos vitamínicos y el indicador de pérdida de peso, referencia para el seguimiento del éxito de la cirugía.

En la actualidad y con el auge de este método invasivo para bajar de peso y reducir las enfermedades concomitantes, es necesario fomentar la creación de grupos interdisciplinarios de excelencia regidos por un consenso nacional. El manejo y seguimiento nutricionales deben evaluarse de nuevo para medir la adherencia y el impacto de las guías de manejo implementadas recientemente.

\section{Agradecimientos}

Agradecemos a la Asociación Colombiana de Nutrición Clínica, y en particular, a las nutricionistas Claudia Angarita, Antonieta Espinosa y Claudia María Carvajal la colaboración e interés en este trabajo.

\section{Conflicto de intereses}

No declara.

\section{Referencias bibliográficas}

1. Organización Mundial de la Salud. Datos sobre la obesidad. Fecha de consulta: $1^{\circ}$ de febrero de 2012. Disponible en: http://www.who.int/features/factfiles/obesity/es/.
2. ENSIN2015. Fecha de consulta: $1^{\circ}$ de febrero de 2018Disponible en: https://www.minsalud.gov.co/Paginas/Gobierno-presentaEncuesta-Nacional-de-Situaci\%C3\%B3n-Nutricional-deColombia-ENSIN-2015.aspx.

3. Sjöström LV. Mortality of severely obese subjects. Am J Clin Nutr. 1992;55:516-23.

4. Mokdad AH, Ford ES, Bowman BA, Dietz WH, Vinicor F, Bales VS, et al. Prevalence of obesity, diabetes, and obesityrelated health risk factors. JAMA. 2003;289:76-9.

5. Eckel RH, Krauss RM. American Heart Association call to action: Obesity as a major risk factor for coronary heart disease. Circulation. 1998;97:2099-100.

6. Calle EE, Thun MJ. Obesity and cancer. Oncogene. 2004;23:6365-78.

7. Office of the Secretary, DoDTRICARE Program: Surgery for morbid obesity. Final Rule Fed Regist. 2011;76:8294-8. Fecha de consulta: 2 de febrero de 2012. Disponible en: http:// edocket.access.gpo.gov/2011/pdf/2011-3207.pdf.

8. Rubio MA, Martínez C, Vidal O, Larrad A, Salas-Salvadó J, Pujol J, et al. Documento de consenso sobre cirugía bariátrica. Rev Esp Obes. 2004;4:223-49.

9. International Federation Diabetes. Bariatric surgical and procedural interventions in the treatment of obese patients with type 2 diabetes. A position statement from the International Diabetes Federation Taskforce on epidemiology and prevention. Fecha de consulta: 8 de febrero de 2012. Disponible en: http://www.diabetesatlas.org.

10. Moreno B, Zugasti A. Cirugía bariátrica: situación actual. Rev Med Univ Navarra. 2004;48:66-71.

11. Catenaccio VA, Hill JO. The obesity epidemic. Chest Med. 2009;30:415-44.

12. Pories WJ, MacDonald KG, Morgan EJ, M K Sinha, G L Dohm, M S Swanson, H A Barakat, P G Khazanie, N LeggettFrazier, S D Long . Surgical treatment of obesity and its effect on diabetes: follow up. Am J Clin Nutr. 1992;55:560-5.

13. Scopinaro N, Gianetta E, Adami GF, Friedman D, Traverso E, Marinari GM, et al. Biliopancreatic diversión for obesity at eight years. Surgery. 1996;119:261-8.

14. Halverson JD. Micronutrient deficiencies after gastric bypass for morbid obesity. Am Surg. 1985;52:594-8.

15. Álvarez-Leite J. Nutrient deficiencies secondary to bariatric surgery. Curr Opin Clin Nutr Metab Care. 2004;7:569-75.

16. Espinosa A. Consenso Colombiano Manejo Nutricional del paciente con cirugía bariátrica y metabólica. RCNM Revista Colombiana de Metabolismo y Nutrición Clínica. 2013;4(1):7-52.

17. Fujiok K. Follow-up of nutritional and metabolic problems after bariatric surgery. Diabetes Care. 2005;28:481-4. 
18. Kelly E. Nutritional considerations after bariatric surgery. Crit Care Nurs Q. 2003;26:133-8.

19. Fried M, Hainer V, Basdevant A, Buchwald H, Deitel M, Finer $\mathrm{N}$, et al. Interdisciplinary European guidelines on surgery of severe obesity. Obes Facts. 2008;1:52-9.

20. Shuster MH, Vázquez JA. Nutritional concerns related to Roux-en-Y gastric bypass: What every clinician needs to know. Crit Care Nurs Q. 2005;28:227-60.

21. Asociación Argentina de Dietistas y Nutricionistas Dietistas. Consenso argentino de nutrición en cirugía bariátrica. Fecha de consulta: 6 de febrero de 2012. Disponible en: http:/ / www. aadynd.org.ar/phocadownload/con_nutricion_ciruj.pdf.

22. Heber D, Greenway FL, Kaplan LM, Livingston E, Salvador J, Still C, Endocrine Society. Endocrine and nutritional management of the post-bariatric surgery patient: An endocrine society clinical practice guideline. Clin Endocrinol Metab. 2010;95:4823-43.

23. McMahon MM, Sarr MG, Clark MM, Knoetgen J 3rd, Service FJ, Laskowski ER, et al. Clinical management after bariatric surgery: Value of a multidisciplinary approach. Mayo Clin Proc. 2006;81(Suppl):S34-45.

24. Bradley DW, Sharma BK. Centers of excellence in bariatric surgery: Design, implementation, and one-year outcomes. Surg Obes Relat Dis. 2006;2:513-7.

25. Velasco N, Häberle S, Alfaro R, Araya A, Lancellotti D. Experiencia en cirugía bariátrica en un hospital regional. Rev Chilena de Cirugía. 2008;60:108-15.

26. Colquitt JL, Picot J, Loveman E, Clegg AJ. Surgery for obesity. Cochrane Database Syst Rev. 2009;(2):CD003641.

27. Fisher B, Schauer P. Medical and surgical options in the treatment of severe obesity. Am J Surg. 2002;184:9-16.

28. Tarnoff M, Kaplan L, Shikora S. An evidenced-based assessment of preoperative weight loss in bariatric surgery. Obes Surg. 2008; 18:1059-61.

29. Mechanick J, Kushner R, Sugerman H, González-Campoy JM, Collazo-Clavell ML, Guven S, et al. Bariatric surgery guidelines. Endocr Pract. 2008;14(Suppl.1):318-36.

30. Kalarchian M, Marcus M. Preoperative weight loss in bariatric surgery. Obes Surg. 2009; 19:539.

31. Rubio A, Moreno C. Implicaciones nutricionales de la cirugía bariátrica sobre el tracto gastrointestinal. Nutr Hosp. 2007;22(Supl.2):124-34.

32. Apovian C, Cummings S, Anderson W, Borud L. Best practice updates for multidisciplinary care in weight loss surgery. Obesity. 2009;17:871-9.

33. Savino P, Zundel N, Carvajal M. Manejo nutricional perioperatorio en pacientes con cirugía bariátrica. Rev Colomb Cir. 2013;28:73-83.

34. Benjaminov O, Beglaibter N, Gindy L, Spivak H, Singer P, Wienberg $\mathrm{M}$, et al. The effect of a low-carbohydrate diet on the non alcoholic fatty liver in morbidly obese patients before bariatric surgery. Surg Endosc. 2007;21:1423-7.

35. Sharma AM, Karmali SH, Birch DW. Reporting weight loss: Is simple better? Obesity. 2010;18:219.

36. Bray GA, Bouchard C, Church TS, Cefalu WT, Greenway FL, Gupta $\mathrm{AK}$, et al. Is it time to change the way we report and discuss weight loss? Obesity. 2009; 17:619-21.

37. Karmali S, Birch DW, Sharma AM. Is it time to abandon excess weight loss in reporting surgical weight loss? Surg Obes Relat Dis. 2009;5:503-6.

38. Wadden TA. Treatment of obesity by moderate and severe caloric restriction. Results of clinical research trials. Ann Intern Med. 1993;119:688-93.

39. Steinbrook R . Surgery for severe obesity. N Engl J Med. 2004;35:1075-9.

40. Parrot J, Frank L, Rabena R, Craggs-Dino L, Isom KA, Greiman L. American Society for Metabolic and Bariatric Surgery Integrated Health Nutritional Guidelines for the Surgical Weight Loss Patient 2016 Update: Micronutrients. Surg Obes Relat Dis. 2017;13(5):727-41.

41. Bock MA. Roux-en-Y gastric bypass: The dietitian's and patient's perspectives. Nutr Clin Pract. 2003;18:141-4.

42. Vásquez C, Morejon E. Repercusión nutricional de la cirugía bariátrica según técnica de Scopinaro: análisis de 40 casos. Nutr Hosp. 2003; 4:189-3.

43. Brolin RE. Bariatric surgery and long-term control of morbid obesity. JAMA. 2002;288:2793-6.

44. Faintuch J, Matsuda M, Cruz ME, Silva MM, Teivelis MP, Garrido AB Jr, et al. Severe protein-calorie malnutrition after bariatric procedures. Obes Surg. 2004;14:175-81.

45. Dolan K, Hatzifotis M, Newbury L, Fielding G. A comparison of laparoscopic adjustable gastric banding and biliopancreatic diversion in superobesity. Obes Surg. 2004;14:165-9.

46. Byrne TK. Complications of surgery for obesity. Surg Clin North Am. 2001;81:1181-93.

47. Kalfarentzos F, Dimakopoulos A, Kehagias I, Loukidi A, Mead $\mathrm{N}$. Vertical banded gastroplasty versus standard or distal Roux- en-Y gastric bypass based on specific selection criteria in the morbidly obese: Preliminary results. Obes Surg. 1999;9: 433-42.

48. Ziegler O, Sirveaux MA, Brunaud L, Reibel N, Quilliot D. Medical follow up after bariatric surgery: Nutritional and drug issues. General recommendations for the prevention and treatment of nutritional deficiencies. Diabetes Metab. 2009;35:544-57.

49. Saris WH, Blair SN, van Baak MA, Eaton SB, Davies PS, Di Pietro L, et al. How much physical activity is enough to prevent unhealthy weight gain? Outcome of the IASO 1st Stock Conference and consensus statement. Obes Rev. 2003;4:101-14.

50. Poirier P, Cornier MA, Mazzone T, Stiles S, Cummings S, Klein $S$, et al. Bariatric surgery and cardiovascular risk factors: A scientific statement from the American Heart Association. Circulation. 2011;123:1683-701. 